\title{
EXTEND MEAN CURVATURE FLOW WITH FINITE INTEGRAL CURVATURE*
}

\author{
HONG-WEI XU ${ }^{\dagger}$, FEI YE ${ }^{\dagger}$, AND EN-TAO ZHAO ${ }^{\dagger}$
}

\begin{abstract}
In this note, we prove that the solution of certain mean curvature flow on a finite time interval $[0, T)$ can be extended over time $T$ if the space-time integration of the mean curvature is finite. Moreover, we show that the condition is optimal in some sense.
\end{abstract}

Key words. Mean curvature flow, maximal existence time, second fundamental form, integral curvature.

AMS subject classifications. 53C44; 53C21.

1. Introduction. Let $M^{n}$ be a complete $n$-dimensional manifold without boundary, and let $F_{t}: M^{n} \rightarrow \mathbb{R}^{n+1}$ be a one-parameter family of smooth hypersurfaces immersed in Euclidean space. We say that $M_{t}=F_{t}(M)$ is a solution of the mean curvature flow if $F_{t}$ satisfies

$$
\left\{\begin{array}{ccc}
\frac{\partial}{\partial t} F(x, t) & = & -H(x, t) \nu(x, t) \\
F(x, 0) & = & F_{0}(x)
\end{array}\right.
$$

where $F(x, t)=F_{t}(x), H(x, t)$ is the mean curvature, $\nu(x, t)$ is the unit outward normal vector, and $F_{0}$ is some given initial hypersurface.

K. Brakke [1] studied the mean curvature flow from the view point of geometric measure theory firstly. For the classical solution of the mean curvature flow, G. Huisken (see $[5,6]$ ) showed that for a smooth complete initial hypersurface with bounded second fundamental form the solution exists on a maximal time interval $[0, T), 0<T \leq \infty$. If the initial hypersurface is closed and convex, he showed in [6] that the mean curvature flow will converge to a round point in finite time. He also proved that if the second fundamental form is uniformly bounded, then the mean curvature flow can be extended.

By a blow up argument, N. Ššum [9] proved that if the Ricci curvature is uniformly bounded on $M \times[0, T)$, then the Ricci flow can be extended over $T$. In [10], B. Wang obtained some integral conditions to extend the Ricci flow. A natural question is that, what is the optimal condition for the mean curvature flow to be extended? By a different method, we investigate the integral conditions to extend the mean curvature flow. We will prove that the mean curvature flow can be extended if the space-time integration of the mean curvature is finite and the second fundamental tensor is bounded from below.

THEOREM 1.1. Let $F_{t}: M^{n} \rightarrow \mathbb{R}^{n+1}(n \geq 3)$ be a solution of the mean curvature flow of closed hypersurfaces on a finite time interval $[0, T)$. If

(1) there is a positive constant $C$ such that $h_{i j} \geq-C$ for $(x, t) \in M \times[0, T)$,

(2) $\|H\|_{\alpha, M \times[0, T)}=\left(\int_{0}^{T} \int_{M_{t}}|H|^{\alpha} d \mu_{t} d t\right)^{\frac{1}{\alpha}}<+\infty$ for some $\alpha \geq n+2$,

*Received May 9, 2010; accepted for publication January 25, 2011. Research supported by the NSFC, Grant No. 11071211, 10771187; the Trans-Century Training Programme Foundation for Talents by the Ministry of Education of China; the Natural Science Foundation of Zhejiang Province, Grant No. 101037; and the China Postdoctoral Science Foundation, Grant No. 20090461379.

$\dagger$ Center of Mathematical Sciences, Zhejiang University, Hangzhou 310027, China (\{xuhw; yf; zhaoet\}@cms.zju.edu.cn). 
then this flow can be extended over time $T$.

When the initial hypersurface is mean convex, we have the following

TheOREM 1.2. Let $F_{t}: M^{n} \rightarrow \mathbb{R}^{n+1}(n \geq 3)$ be a solution of the mean curvature flow of closed hypersurfaces on a finite time interval [0,T). If

(1) $H>0$ at $t=0$,

(2) $\|H\|_{\alpha, M \times[0, T)}=\left(\int_{0}^{T} \int_{M_{t}}|H|^{\alpha} d \mu_{t} d t\right)^{\frac{1}{\alpha}}<+\infty$ for some $\alpha \geq n+2$, then this flow can be extended over time $T$.

The following example shows that the condition $\alpha \geq n+2$ in Theorems 1.1 and 1.2 are optimal.

EXAMPLE. Set $\mathbb{S}^{n}=\left\{x \in \mathbb{R}^{n+1}: \sum_{i=1}^{n} x_{i}^{2}=1\right\}$. Let $F$ be the standard isometric embedding of $\mathbb{S}^{n}$ into $\mathbb{R}^{n+1}$. It is clear that $F(t)=\sqrt{1-2 n t} F$ is the solution to the mean curvature flow, where $T=\frac{1}{2 n}$ is the maximal existence time. By a simple computation, we have $g_{i j}(t)=(1-2 n t) g_{i j}, H(t)=\frac{n}{\sqrt{1-2 n t}}$ and $h_{i j}(t) \geq 0$. Hence

$$
\begin{aligned}
\|H\|_{\alpha, M \times[0, T)} & =\left(\int_{0}^{T} \int_{M_{t}}|H|^{\alpha} d \mu_{t} d t\right)^{\frac{1}{\alpha}} \\
& =C_{1}\left(\int_{0}^{T}(T-t)^{\frac{n-\alpha}{2}} d t\right)^{\frac{1}{\alpha}},
\end{aligned}
$$

where $C_{1}$ is a positive constant. It follows that

$$
\|H\|_{\alpha, M \times[0, T)} \begin{cases}=\infty, & \text { for } \alpha \geq n+2 \\ <\infty, & \text { for } \alpha<n+2 .\end{cases}
$$

This implies that the condition $\alpha \geq n+2$ in Theorems 1.1 and 1.2 are optimal.

2. An upper bound of the mean curvature by its $L^{n+2}$-norm. Let $F$ : $M^{n} \rightarrow \mathbb{R}^{n+1}$ be a compact immersed hypersurface. Denote by $g=\left\{g_{i j}\right\}$ the induced metric, $A=\left\{h_{i j}\right\}$ the second fundamental form, $\nabla$ the induced Levi-Civita connection and $\triangle$ the induced Laplacian. The volume form on $M$ is $d \mu=\sqrt{\operatorname{det}\left(g_{i j}\right)} d x$, and the mean curvature $H$ is the trace of the second fundamental form.

In this section we obtain an inequality relating the mean curvature and its $L^{n+2}$-norm in the space-time. We first recall some evolution equations (see $[3,14]$ ).

LEMMA 2.1. Along the mean curvature flow we have the following evolution equations.

$$
\begin{aligned}
& \frac{\partial}{\partial t} g_{i j}=-2 H h_{i j}, \\
& \frac{\partial}{\partial t} d \mu_{t}=-H^{2} d \mu_{t}, \\
& \frac{\partial}{\partial t} H=\triangle H+|A|^{2} H, \\
& \frac{\partial}{\partial t}|A|^{2}=\triangle|A|^{2}-2|\nabla A|^{2}+2|A|^{4} .
\end{aligned}
$$


The following Sobolev inequality can be found in [8] and [12].

LEMMA 2.2. Let $M^{n}$ be an $n$-dimensional $(n \geq 3)$ closed submanifold of a Riemannian manifold $N^{n+p}$ with codimension $p \geq 1$. Suppose that the sectional curvature of $N^{n+p}$ is non-positive. Then for any $s \in(0,+\infty)$ and $f \in C^{1}(M)$ such that $f \geq 0$,

$$
\int_{M}|\nabla f|^{2} d \mu \geq \frac{(n-2)^{2}}{4(n-1)(1+s)}\left[\frac{1}{C^{2}(n)}\left(\int_{M} f^{\frac{2 n}{n-2}} d \mu\right)^{\frac{n-2}{n}}-H_{0}^{2}\left(1+\frac{1}{s}\right) \int_{M} f^{2} d \mu\right]
$$

where $H_{0}=\max _{x \in M}|H|, C(n)=\frac{2^{n}(1+n)^{\left(1+\frac{1}{n}\right)}}{(n-1) \sigma_{n}}$, and $\sigma_{n}$ is the volume of the unit ball in $\mathbb{R}^{n+1}$.

The following estimate is very useful in the proofs of our theorems.

THEOREM 2.3. Suppose that $F_{t}: M^{n} \rightarrow \mathbb{R}^{n+1}(n \geq 3)$ is a mean curvature flow solution for $t \in\left[0, T_{0}\right]$, and the second fundamental form is uniformly bounded on the time interval $\left[0, T_{0}\right]$. Then

$$
\max _{(x, t) \in M \times\left[\frac{T_{0}}{2}, T_{0}\right]} H^{2}(x, t) \leq C_{2}\left(\int_{0}^{T_{0}} \int_{M_{t}}|H|^{n+2} d \mu_{t} d t\right)^{\frac{2}{n+2}}
$$

where $C_{2}$ is a constant depending on $n, T_{0}$ and $\sup _{(x, t) \in M \times\left[0, T_{0}\right]}|A|$.

Proof. The evolution equation of $H^{2}$ is

$$
\frac{\partial}{\partial t} H^{2}=\triangle H^{2}-2|\nabla H|^{2}+2|A|^{2} H^{2} \text {. }
$$

Since $A$ is bounded, we obtain the following estimate from (1) that

$$
\frac{\partial}{\partial t} H^{2} \leq \triangle H^{2}+\beta H^{2}
$$

where $\beta$ is a constant depending only on $\sup _{(x, t) \in M \times\left[0, T_{0}\right]}|A|$.

Denoting $f=H^{2}$, from the inequality in (2) and the evolution equation of the volume form in Lemma 2.1, we obtain that for any $p \geq 2$,

$$
\begin{aligned}
\frac{\partial}{\partial t} \int_{M_{t}} f^{p} d \mu_{t} & =\int_{M_{t}} p f^{p-1} \frac{\partial}{\partial t} f d \mu_{t}-\int_{M_{t}} f^{p+1} d \mu_{t} \\
& \leq \int_{M_{t}} p f^{p-1}(\triangle f+\beta f) d \mu_{t} \\
& =-\frac{4(p-1)}{p} \int_{M_{t}}\left|\nabla f^{\frac{p}{2}}\right|^{2} d \mu_{t}+\beta p \int_{M_{t}} f^{p} d \mu_{t} .
\end{aligned}
$$

Thus

$$
\frac{\partial}{\partial t} \int_{M_{t}} f^{p} d \mu_{t}+\frac{4(p-1)}{p} \int_{M_{t}}\left|\nabla f^{\frac{p}{2}}\right|^{2} d \mu_{t} \leq \beta p \int_{M_{t}} f^{p} d \mu_{t}
$$


For any $0<\tau<\tau^{\prime}<T_{0}$, define a function $\psi$ on $\left[0, T_{0}\right]$ :

$$
\psi(t)= \begin{cases}0 & 0 \leq t \leq \tau \\ \frac{t-\tau}{\tau^{\prime}-\tau} & \tau \leq t \leq \tau^{\prime} \\ 1 & \tau^{\prime} \leq t \leq T_{0}\end{cases}
$$

Then by (3) we have

$$
\begin{aligned}
\frac{\partial}{\partial t}\left(\psi \int_{M_{t}} f^{p} d \mu_{t}\right)= & \psi^{\prime} \int_{M_{t}} f^{p} d \mu_{t}+\psi \frac{\partial}{\partial t}\left(\int_{M_{t}} f^{p} d \mu_{t}\right) \\
\leq & \psi^{\prime} \int_{M_{t}} f^{p} d \mu_{t}+\psi\left(-\frac{4(p-1)}{p} \int_{M_{t}}\left|\nabla f^{\frac{p}{2}}\right|^{2} d \mu_{t}\right. \\
& \left.+\beta p \int_{M_{t}} f^{p} d \mu_{t}\right) .
\end{aligned}
$$

For any $t \in\left[\tau^{\prime}, T_{0}\right]$, integrating both sides of the inequality in $(4)$ on $[\tau, t]$ we get

(5) $\quad \int_{M_{t}} f^{p} d \mu_{t}+\frac{4(p-1)}{p} \int_{\tau^{\prime}}^{t} \int_{M_{t}}\left|\nabla f^{\frac{p}{2}}\right|^{2} d \mu_{t} d t \leq\left(\beta p+\frac{1}{\tau^{\prime}-\tau}\right) \int_{\tau}^{T_{0}} \int_{M_{t}} f^{p} d \mu_{t} d t$.

For the integral $\int_{\tau^{\prime}}^{T_{0}} \int_{M_{t}} f^{p\left(1+\frac{2}{n}\right)} d \mu_{t} d t$, by Schwarz inequality and Sobolev inequality in Lemma 2.2, we have

$$
\begin{aligned}
\int_{\tau^{\prime}}^{T_{0}} \int_{M_{t}} f^{p\left(1+\frac{2}{n}\right)} d \mu_{t} d t \leq & \int_{\tau^{\prime}}^{T_{0}}\left(\int_{M_{t}} f^{p} d \mu_{t}\right)^{\frac{2}{n}}\left(\int_{M_{t}} f^{\frac{n p}{n-2}} d \mu_{t}\right)^{\frac{n-2}{n}} d t \\
\leq & \max _{t \in\left[\tau^{\prime}, T_{0}\right]}\left(\int_{M_{t}} f^{p} d \mu_{t}\right)^{\frac{2}{n}} \int_{\tau^{\prime}}^{T_{0}}\left(\int_{M_{t}} f^{\frac{n p}{n-2}} d \mu_{t}\right)^{\frac{n-2}{n}} d t \\
\leq & \left(\beta p+\frac{1}{\tau^{\prime}-\tau}\right)^{\frac{2}{n}}\left(\int_{\tau}^{T_{0}} \int_{M_{t}} f^{p} d \mu_{t} d t\right)^{\frac{2}{n}} \\
& \times \int_{\tau^{\prime}}^{T_{0}}\left[\frac{4(n-1) C^{2}(n)(1+s)}{(n-2)^{2}} \int_{M_{t}}\left|\nabla f^{\frac{p}{2}}\right|^{2} d \mu_{t}\right. \\
& \left.+\frac{n}{2} \beta C^{2}(n)\left(1+\frac{1}{s}\right) \int_{M_{t}} f^{p} d \mu_{t}\right] d t .
\end{aligned}
$$


For the third factor on the right hand side, we have from (5)

$$
\begin{aligned}
& \int_{\tau^{\prime}}^{T_{0}}\left[\frac{4(n-1) C^{2}(n)(1+s)}{(n-2)^{2}} \int_{M_{t}}\left|\nabla f^{\frac{p}{2}}\right|^{2} d \mu_{t}+\frac{n}{2} \beta C^{2}(n)\left(1+\frac{1}{s}\right) \int_{M_{t}} f^{p} d \mu_{t}\right] d t \\
\leq & \frac{4(n-1) C^{2}(n)(1+s)}{(n-2)^{2}} \int_{\tau^{\prime}}^{T_{0}} \int_{M_{t}}\left|\nabla f^{\frac{p}{2}}\right|^{2} d \mu_{t} d t \\
& +\frac{n}{2} \beta C^{2}(n)\left(1+\frac{1}{s}\right) \int_{\tau^{\prime}}^{T_{0}}\left[\left(\beta p+\frac{1}{\tau^{\prime}-\tau}\right) \int_{\tau}^{T_{0}} \int_{M_{t}} f^{p} d \mu_{t} d t\right] d t \\
\leq & \frac{(n-1) C^{2}(n) p(1+s)}{(n-2)^{2}(p-1)}\left(\beta p+\frac{1}{\tau^{\prime}-\tau}\right) \int_{\tau}^{T_{0}} \int_{M_{t}} f^{p} d \mu_{t} d t \\
& +\frac{n}{2} \beta C^{2}(n) T_{0}\left(1+\frac{1}{s}\right)\left(\beta p+\frac{1}{\tau^{\prime}-\tau}\right) \int_{\tau}^{T_{0}} \int_{M_{t}} f^{p} d \mu_{t} d t \\
= & {\left[\frac{(n-1) C^{2}(n) p(1+s)}{(n-2)^{2}(p-1)}+\frac{n}{2} \beta C^{2}(n) T_{0}\left(1+\frac{1}{s}\right)\right]\left(\beta p+\frac{1}{\tau^{\prime}-\tau}\right) \int_{\tau}^{T_{0}} \int_{M_{t}} f^{p} d \mu_{t} d t . }
\end{aligned}
$$

Hence

$$
\begin{aligned}
& \int_{\tau^{\prime}}^{T_{0}} \int_{M_{t}} f^{p\left(1+\frac{2}{n}\right)} d \mu_{t} d t \\
\leq & {\left[\frac{(n-1) C^{2}(n) p(1+s)}{(n-2)^{2}(p-1)}+\frac{n}{2} \beta C^{2}(n) T_{0}\left(1+\frac{1}{s}\right)\right] } \\
& \times\left(\beta p+\frac{1}{\tau^{\prime}-\tau}\right)^{1+\frac{2}{n}}\left(\int_{\tau}^{T_{0}} \int_{M_{t}} f^{p} d \mu_{t} d t\right)^{1+\frac{2}{n}} .
\end{aligned}
$$

Put $L(p, t)=\int_{t}^{T_{0}} \int_{M_{t}} f^{p} d \mu_{t} d t, s=\frac{\left[\frac{2}{n}(p-1) T_{0} \beta\right]^{\frac{1}{2}}(n-2)}{[n(p-1)]^{\frac{1}{2}}}$, and $D_{n, q}=\frac{[(n-1) p]^{\frac{1}{2}} C(n)}{(n-2)(p-1)^{\frac{1}{2}}}$. The inequality in (6) can be rewritten as

$$
L\left(p\left(1+\frac{2}{n}\right), \tau^{\prime}\right) \leq D_{n, q}^{2}\left(\beta p+\frac{1}{\tau^{\prime}-\tau}\right)^{1+\frac{2}{n}} L(p, \tau)^{1+\frac{2}{n}}
$$

Now let $\mu=1+\frac{2}{n}, p_{k}=\frac{n+2}{2} \mu^{k}$ and $\tau_{k}=\left(1-\frac{1}{\mu^{k+1}}\right) t$. Then from (7) we obtain

(8)L( $\left.p_{k+1}, \tau_{k+1}\right)^{\frac{1}{p_{k+1}}} \leq D_{n, \frac{n+2}{2}}^{\sum_{i=0}^{k} \frac{2}{p_{i+1}}}\left(\frac{(n+2) \beta}{2}+\frac{n+2}{2 t}\right)^{\sum_{i=0}^{k} \frac{1}{p_{i}}} \mu^{\sum_{i=0}^{k} \frac{i}{p_{i}}} L\left(p_{0}, \tau_{0}\right)^{\frac{2}{n+2}}$.

As $k \rightarrow+\infty$, we conclude from (8) that

(9) $f(x, t) \leq D_{n, \frac{n+2}{2}}^{\frac{2 n}{n+2}}\left(1+\frac{2}{n}\right)^{\frac{n}{2}}\left(\frac{n+2}{2} \beta+\frac{n+2}{2 t}\right)\left(\int_{0}^{T_{0}} \int_{M_{t}} f^{\frac{n+2}{2}} d \mu_{t} d t\right)^{\frac{2}{n+2}}$.

Therefore, for any $(x, t) \in M \times\left[\frac{T_{0}}{2}, T_{0}\right]$, we get from (9)

$$
H^{2}(x, t) \leq C_{2}\left(\int_{0}^{T_{0}} \int_{M_{t}}|H|^{n+2} d \mu_{t} d t\right)^{\frac{2}{n+2}}
$$


where $C_{2}$ is a constant depending on $n, T_{0}$ and $\sup _{(x, t) \in M \times\left[0, T_{0}\right]}|A|$. Since $(x, t) \in$ $M \times\left[\frac{T_{0}}{2}, T_{0}\right]$ is arbitrary, it follows from (10) that

$$
\max _{(x, t) \in M \times\left[\frac{T_{0}}{2}, T_{0}\right]} H^{2}(x, t) \leq C_{2}\left(\int_{0}^{T_{0}} \int_{M_{t}}|H|^{n+2} d \mu_{t} d t\right)^{\frac{2}{n+2}},
$$

which is desired.

3. Mean curvature flow with finite total mean curvature. We are now in a position to prove our theorems.

Proof of Theorem 1.1. We only need to prove the theorem for $\alpha=n+2$ since by Hölder's inequality, $\|H\|_{\alpha, M \times[0, T)}<\infty$ for $\alpha>n+2$ implies $\|H\|_{n+2, M \times[0, T)}<\infty$. We argue by contradiction.

Suppose that the solution of the mean curvature flow cannot be extended over $T$. Then $A$ becomes unbounded as $t \rightarrow T$. Since $h_{i j} \geq-C$, we get $\sum_{i, j}\left(h_{i j}+C\right)^{2} \leq$ $C_{3}\left[\operatorname{tr}\left(h_{i j}+C\right)\right]^{2}$, where $C_{3}$ is a constant depending only on $n$. On one hand, $|A|^{2}$ is unbounded implies that $\sum_{i, j}\left(h_{i j}+C\right)^{2}$ is unbounded. On the other hand,

$$
\left[\operatorname{tr}\left(h_{i j}+C\right)\right]^{2}=(H+n C)^{2}=H^{2}+2 n C H+n^{2} C^{2} .
$$

Thus $H^{2}$ is unbounded. Namely,

$$
\sup _{(x, t) \in M \times[0, T)} H^{2}(x, t)=\infty .
$$

Choose an increasing time sequence $t^{(i)}, i=1,2, \cdots$, such that $\lim _{i \rightarrow \infty} t^{(i)}=T$. We take a sequence of points $x^{(i)} \in M$ satisfying

$$
H^{2}\left(x^{(i)}, t^{(i)}\right)=\max _{(x, t) \in M \times\left[0, t^{(i)}\right)} H^{2}(x, t) .
$$

Then $\lim _{i \rightarrow \infty} H^{2}\left(x^{(i)}, t^{(i)}\right)=\infty$.

Putting $Q^{(i)}=H^{2}\left(x^{(i)}, t^{(i)}\right)$, we have $\lim _{i \rightarrow \infty} Q^{(i)}=\infty$. This together with $\lim _{i \rightarrow \infty} t^{(i)}=T>0$ implies that there exists a positive integer $i_{0}$ such that $Q^{(i)} t^{(i)} \geq 1$ and $Q^{(i)} \geq 1$ for $i \geq i_{0}$. For $i \geq i_{0}$ and $t \in[0,1]$, we define $F^{(i)}(t)=\left(Q^{(i)}\right)^{\frac{1}{2}} F\left(\frac{t-1}{Q^{(i)}}+t^{(i)}\right)$. Then the metric on $M$ induced by $F^{(i)}(t)$ is $g^{(i)}(t)=Q^{(i)} g\left(\frac{t-1}{Q^{(i)}}+t^{(i)}\right)$, and $F^{(i)}(t): M^{n} \rightarrow \mathbb{R}^{n+1}$ is still a solution of the mean curvature flow on $t \in[0,1]$. Since $F_{t}$ satisfies $h_{i j} \geq-C$ for $(x, t) \in M \times[0, T)$, we have

$$
\begin{gathered}
H_{(i)}^{2}(x, t) \leq 1 \text { on } M \times[0,1], \\
h_{j k}^{(i)} \geq-\frac{C}{\sqrt{Q^{(i)}}} \text { on } M \times[0,1],
\end{gathered}
$$

where $H_{(i)}$ and $A^{(i)}=h_{j k}^{(i)}$ are the mean curvature and the second fundamental form of $F^{(i)}(t)$, respectively. The inequality in (11) gives that $h_{j k}^{(i)}+\frac{C}{\sqrt{Q^{(i)}}} \geq 0$. Hence

$$
h_{j k}^{(i)}+\frac{C}{\sqrt{Q^{(i)}}} \leq \operatorname{tr}\left(h_{j k}^{(i)}+\frac{C}{\sqrt{Q^{(i)}}}\right) \leq H_{(i)}+\frac{n C}{\sqrt{Q^{(i)}}} .
$$


The inequality in (12) implies that $h_{j k}^{(i)} \leq H_{(i)}+\frac{(n-1) C}{\sqrt{Q^{(i)}}}$. Since $Q^{(i)} \geq 1$ when $i \geq i_{0}$, it follows that $\left|A^{(i)}\right| \leq C_{4}$ for $i \geq i_{0}$ and $t \in[0,1]$, where $C_{4}$ is a positive constant independent of $i$.

Set $\left(M^{(i)}, g^{(i)}(t), x^{(i)}\right)=\left(M, Q^{(i)} g\left(\frac{t-1}{Q^{(i)}}+t^{(i)}\right), x^{(i)}\right), t \in[0,1]$. From [2] we know that there is a subsequence of $\left(M^{(i)}, g^{(i)}(t), x^{(i)}\right)$ converges to a Riemannian manifold $(\widetilde{M}, \widetilde{g}(t), \widetilde{x})$, and the corresponding subsequence of immersions $F^{(i)}(t)$ converges to an immersion $\widetilde{F}(t): \widetilde{M} \rightarrow \mathbb{R}^{n+1}$. Also, it follows from Theorem 2.3 that for $i \geq i_{0}$,

$$
\max _{(x, t) \in M^{(i)} \times\left[\frac{1}{2}, 1\right]} H_{(i)}^{2}(x, t) \leq C_{5}\left(\int_{0}^{1} \int_{M_{t}}|H|_{(i)}^{n+2} d \mu_{g^{(i)}(t)} d t\right)^{\frac{2}{n+2}}
$$

where $C_{5}$ is a constant independent of $i$. Hence

$$
\begin{aligned}
\max _{(x, t) \in \widetilde{M} \times\left[\frac{1}{2}, 1\right]} \widetilde{H}^{2}(x, t) & \leq \lim _{i \rightarrow \infty} C_{5}\left(\int_{0}^{1} \int_{M_{t}}|H|_{(i)}^{n+2} d \mu_{g^{(i)}(t)} d t\right)^{\frac{2}{n+2}} \\
& \leq \lim _{i \rightarrow \infty} C_{5}\left(\int_{t^{(i)}}^{t^{(i)}+\left(Q^{(i)}\right)^{-1}} \int_{M_{t}}|H|_{(i)}^{n+2} d \mu_{t} d t\right)^{\frac{2}{n+2}} \\
& =0 .
\end{aligned}
$$

The equality in (13) holds because $\int_{0}^{T} \int_{M_{t}} H^{n+2} d \mu d t<+\infty$ and $\lim _{i \rightarrow \infty}\left(Q^{(i)}\right)^{-1}=0$. However, according to the choice of the points, we have

$$
\widetilde{H}^{2}(\widetilde{x}, 1)=\lim _{i \rightarrow \infty} H_{(i)}^{2}\left(x^{(i)}, 1\right)=1 .
$$

This is a contradiction. We complete the proof of Theorem 1.1.

With a similar method, we can prove Theorem 1.2.

Proof of Theorem 1.2. Since $H>0$ at $t=0$, there exists a positive constant $C_{6}$ such that $|A|^{2} \leq C_{6} H^{2}$. The evolution equation of $H$ in Lemma 2.1 implies that $H>0$ is preserved along the mean curvature flow. By [7] we have the following evolution equation of $\frac{|A|^{2}}{H^{2}}$

(14) $\frac{\partial}{\partial t}\left(\frac{|A|^{2}}{H^{2}}\right)=\triangle\left(\frac{|A|^{2}}{H^{2}}\right)+\frac{2}{H}\left\langle\nabla H, \nabla\left(\frac{|A|^{2}}{H^{2}}\right)\right\rangle-\frac{2}{H^{4}}\left|H \nabla_{i} h_{j k}-\nabla_{i} H \cdot h_{j k}\right|^{2}$.

Using the maximum principle, we obtain from (14) that $|A|^{2} \leq C_{6} H^{2}$ is preserved along the mean curvature flow.

It is sufficient to prove the theorem for $\alpha=n+2$. We still argue by contradiction. Suppose that the solution of the mean curvature flow cannot be extended over time $T$. Then $|A|^{2}$ is unbounded as $t \rightarrow T$. This implies that $H^{2}$ is also unbounded since $|A|^{2} \leq C_{6} H^{2}$. Let $\left(x^{(i)}, t^{(i)}\right), Q^{(i)}, F^{(i)}(t), g^{(i)}(t)$ and $(\widetilde{M}, \tilde{g}(t), \tilde{x})$ be the same as in the proof of Theorem 1.1. Let $A^{(i)}$ and $H_{(i)}$ be the second fundamental form and mean curvature of the immersion $F^{(i)}(t)$, respectively. Then we have $\left|A^{(i)}\right|^{2} \leq C_{6}\left|H_{(i)}\right|^{2}$ for $(x, t) \in M \times[0,1]$, which implies that $A^{(i)}$ is bounded by a constant independent of $i$ 
for $t \in[0,1]$. It follows from Theorem 2.3 that

$$
\max _{(x, t) \in M^{(i)} \times\left[\frac{1}{2}, 1\right]} H_{(i)}^{2}(x, t) \leq C_{7}\left(\int_{0}^{1} \int_{M_{t}}|H|_{(i)}^{n+2} d \mu_{g^{(i)}(t)} d t\right)^{\frac{2}{n+2}}
$$

where $C_{7}$ is a constant independent of $i$. By an argument similar to the proof of Theorem 1.1, we get a contradiction which completes the proof of Theorem 1.2.

Finally we would like to propose the following

Open Question. Can one generalize Theorems 1.1 and 1.2 to the case where $F_{t}$ is the solution of mean curvature flow of closed submanifolds in a Riemannian manifold?

\section{REFERENCES}

[1] K. Brakke, The Motion of a surface by its mean curvature, Princeton Univ. Press, Princeton, NJ, 1978.

[2] J. Y. Chen And W. Y. He, A note on singular time of mean curvature flow, Math. Z., 266 (2010), pp. 921-931.

[3] B. Chow, P. Lu AND L. Ni, Hamilton's Ricci flow, Graduate Studies in Mathematics 77, Science Press, New York, 2006.

[4] X. Z. DaI, G. F. Wei And R. G. Ye, Smoothing Riemannian metrics with Ricci curvature bounds, Manu. Math., 90 (1996), pp. 49-61.

[5] K. Ecker And G. Huisken, Interior estimates for hypersurfaces moving by mean curvature, Invent. Math., 105 (1991), pp. 547-569.

[6] G. Huisken, Flow by mean curvature of convex surfaces into spheres, J. Differential Geom., 20 (1984), pp. $237-266$.

[7] G. Huisken and C. Sinestrari, Mean curvature flow singularities for mean convex surfaces, Calc. Var. Partial Differential Equations, 8 (1999), pp. 1-14.

[8] D. Hoffman And J. Spruck, Sobolev and isoperimetric inequalities for Riemannian submanifolds, Comm. Pure Appl. Math., 27 (1974), pp. 715-727.

[9] N. ŠEšum, Curvature tensor under the Ricci flow, Amer. J. Math., 127 (2005), pp. 1315-1324.

[10] B. WANG, On the conditions to extend Ricci flow, International Math. Res. Notices, vol. 2008.

[11] M. T. WANG, Mean curvature flow of surfaces in Einstein four-manifolds, J. Differential Geom., 57 (2001), pp. 301-338.

[12] H. W. XU AND J. R. GU, A general gap theorem for submanifolds with parallel mean curvature in $\mathbb{R}^{n+p}$, Comm. Anal. Geom., 15 (2007), pp. 175-193.

[13] H. W. Xu And E. T. Zhaо, Deforming a hypersurface in $\mathbb{R}^{n+1}$ with small total curvature, Preprint.

[14] X. P. ZHU, Lectures on mean curvature flows, Studies in Advanced Mathematics 32, International Press, Somerville, 2002. 